\title{
CXCR4/SDF1 mediate hypoxia induced chondrosarcoma cell invasion through ERK signaling and increased MMP1 expression
}

\author{
Xiaojuan Sun, Lei Wei, Qian Chen, Richard M Terek
}

\begin{abstract}
Background: Chondrosarcoma is a disease that does not respond to conventional cytotoxic chemotherapy and expression of MMP1 is a marker for a poor prognosis. The mechanism of increased MMP1 expression in chondrosarcoma is not completely known. Our goal is to identify molecular pathways that could serve as therapeutic targets. Chondrosarcoma become hypoxic as they grow, are capable of eliciting an angiogenic response, and typically metastasize to the lungs. The present study determined the effect of hypoxia and specifically HIF-1a on expression of CXCR4 and MMP1 and their role in chondrosarcoma cell invasion.

Results: CXCR4 and its ligand, SDF1, are upregulated in primary chondrosarcoma tumors compared to normal articular cartilage, and CXCR4 was upregulated in chondrosarcoma cell line JJ compared to normal chondrocytes. Hypoxia and specifically HIF-1a increased CXCR4 and MMP1 expression in JJ cell line and chondrosarcoma invasion in vitro. The hypoxia mediated increase in MMP1 expression and chondrosarcoma invasion could be inhibited by siRNA directed at HIF-1a or CXCR4, the CXCR4 inhibitor AMD3100, as well as with ERK inhibitor U0126 and ERK SiRNA.

Conclusions: Chondrosarcoma cell invasion is increased by hypoxia induced expression of CXCR4 and MMP1 and is mediated by HIF-1a and ERK. Both invasion and MMP1 can be inhibited with CXCR4 blockade, suggesting that CXCR4/SDF1 signaling may be a therapeutic target for chondrosarcoma.
\end{abstract}

\section{Background}

Chondrosarcoma is the second most common primary malignant bone. It is a rare disease with a poor prognosis, usually occurs in adults, and the cure rate for this disease has not improved over the last several decades $[1,2]$. For high grade tumors the cure rate has remained at $10-25 \%[3]$. The treatment for chondrosarcoma is surgical resection; chemotherapy and radiation therapy are not ordinarily used since chondrosarcoma are resistant to these adjuvant modalities. In contrast to chondrosarcoma, osteosarcoma, which usually occurs in adolescents, is sensitive to chemotherapy and the cure rate has increased from $20 \%$ to $75 \%$ with the advent of multiagent chemotherapy. However, in patients with either tumor, the majority of those who are not cured succumb to lung metastases. Our efforts are directed at

\footnotetext{
* Correspondence: Richard_Terek@Brown.edu

Department of Orthopaedics, Warren Alpert Medical School of Brown University and Rhode Island Hospital, Providence RI, USA
}

elucidating the mechanisms of chondrosarcoma invasion and metastasis.

Invasion, angiogenesis, migration, and metastasis are intertwined processes regulated by overlapping molecular pathways. Chemokines and their receptors compose one such pathway and are involved with cell trafficking, migration, and proliferation. There are four groups of chemokine receptors: $\mathrm{C}, \mathrm{CC}, \mathrm{CXC}$, and $\mathrm{CX} 3 \mathrm{C}$. Chemokine receptor four (CXCR4) is a seven-transmembrane G-protein-coupled receptor, whose activation leads to intracellular signaling cascades. CXCR4 is expressed in dendritic cells, naïve T cells, NK cells, and monocytes and is also the chemokine receptor most commonly expressed in tumors. Within normal cells chemokine receptors are important in immune cell function and migration of stem cells to sites of injury. Within tumor cells, chemokine receptor expression is related to development of metastases preferentially to sites with expression of the corresponding chemokine[4]. The ligand for 
CXCR4 is the chemokine stromal cell derived factor one (SDF1) which is expressed in the lung and other sites of metastases. CXCR4/SDF1 also indirectly promotes tumor metastasis by mediating proliferation and migration of tumor cells and enhancing tumor-associated angiogenesis [5]. The expression of chemokine receptors has been mostly investigated in carcinoma and increased levels of expression have been found in breast, gastric, colorectal, and lung cancer. CXCR4 expression has also been studied in melanoma, chondrosarcoma, and osteosarcoma. In the latter expression of CXCR4 correlates with overall survival, event-free survival, and metastasis free survival [6] For review see [7,8].

Another factor that drives aggressive behavior in cancer is hypoxia. Hypoxia is a signal that develops as tumors outgrow their blood supply and results in a large number of adaptive changes aimed at surviving in the hypoxic environment as well as correcting the oxygen deficit. HIF-1 is a dimeric transcription factor composed of HIF1 alpha and beta subunits. HIF-1 protein levels increase as a result of decreased degradation of the oxygen sensitive subunit HIF-1alpha. HIF-1 modulates changes in gene expression during hypoxia. One of the better characterized phenotypic changes induced by hypoxia is angiogenesis, largely mediated by HIF-1 and vascular endothelial growth factor (VEGF) which increases vessel ingrowth from surrounding tissue into the tumor. Our prior work has shown that grades II and III chondrosarcoma express higher levels of HIF-1 and VEGF than benign and grade I cartilage tumors[9] Grades II and III chondrosarcoma are the tumors that metastasize and have poor survival. Hypoxia is also known to increase CXCR4 expression in other systems [10].

Tissue invasion by tumor cells and tumor induced blood vessels also requires matrix metalloproteinases. Specific tumors preferentially express different MMPs. In chondrosarcoma, MMP1 is the dominant metalloproteinase that is expressed and is a marker for poor prognosis [11]. However, the mechanisms of increased MMP1 expression in chondrosarcoma are incompletely understood.

Therefore, we investigated the expression of CXCR4 in normal chondrocytes, normal cartilage, chondrosarcoma tissue, and chondrosarcoma cells (CS) and hypothesized that CXCR4 is overexpressed in chondrosarcoma, is upregulated by hypoxia and specifically by HIF-1, and increases the invasive phenotype by increasing expression of MMP1.

\section{Results}

SDF1 and CXCR4 expression are increased in primary chondrosarcoma

As a first step in evaluating the potential role of SDF1 and CXCR4 in chondrosarcoma biology, we analyzed primary chondrosarcoma tissue and articular cartilage for expression of mRNA and protein for these genes using qRT-PCR and Western blotting. We found that the median CXCR4 and SDF1 mRNA levels were 109 compared to 3 and 117 compared to 2 in the tumors compared to normal tissue (Fig. 1A and 1B), and the expression of CXCR4 correlated with tumor grade $(\mathrm{p}<$ 0.001, ANOVA). Western blot of CXCR4 expression for a subset of primary tumors and normal cartilage showed similar results. (Fig. 1C).

\section{Effect of hypoxia on endogenous CXCR4 expression in} chondrosarcoma cell line

In chondrosarcoma cell line, the endogenous CXCR4 mRNA level was increased 6 fold compared to chondrocytes (Fig. 2A). Since tumors become hypoxic as they grow, and hypoxia increases expression of genes related to the malignant phenotype, we evaluated the expression of CXCR4 under hypoxic conditions. CXCR4 mRNA expression in JJ cells showed a progressive increase during hypoxia $\left(2 \% \mathrm{O}_{2}\right)$ that reached 16 fold after $48 \mathrm{~h}$ (Fig. $2 \mathrm{~B}, \mathrm{C})$. Western blot confirmed the qRT-PCR results (Fig. 2D).

\section{HIF-1a regulates CXCR4 expression}

In order to assess if Hif-1a specifically mediates the increase in CXCR4 expression seen during hypoxia, HIF-1a transfection was performed. CXCR4 mRNA level increased by 3 fold relative to the empty vector control (Fig. 3A). Conversely, knockdown of Hif-1a with specific siRNA in JJ cultured in hypoxia decreased CXCR4 mRNA by $56 \%$ (Fig. 3C, D) and had the expected effect on Hif-1a expression (Fig. 3B, D). Western Blot showed the expressions of CXCR4 and Hif1a were reduced after Hif-1a knockdown during hypoxia. (Fig. 3E).

Effect of hypoxia, HIF-1a and CXCR4 knockdown, and CXCR4 blockade on invasion

To test whether overexpression of CXCR4 drives chondrosarcoma cell metastasis, an in vitro cell invasion assay was performed. When cells were cultured in hypoxia and an SDF1 gradient, cell invasion increased 2 fold compared to normoxia, $\mathrm{p}<0.05$. Knockdown of Hif-1a or CXCR4 with specific siRNA completely blocked this increase in invasion that occurs during hypoxic culture (Fig. 4). Similarly, when the cells were pretreated with the CXCR4 inhibitor AMD3100, the hypoxia and SDF1 mediated increase in cell invasion was blocked, whereas AMD3100 had no effect during normoxia (Fig. 5).

Hypoxia and CXCR4 signaling increase MMP1 expression and activity

Cell invasion is in part mediated by matrix metalloproteinases. Figure 6 shows the effects of hypoxia and CXCR4 stimulation with SDF-1 or CXCR4 blockade with AMD3100 on MMP1 mRNA expression and secreted active MMP1 protein. Hypoxia increased 


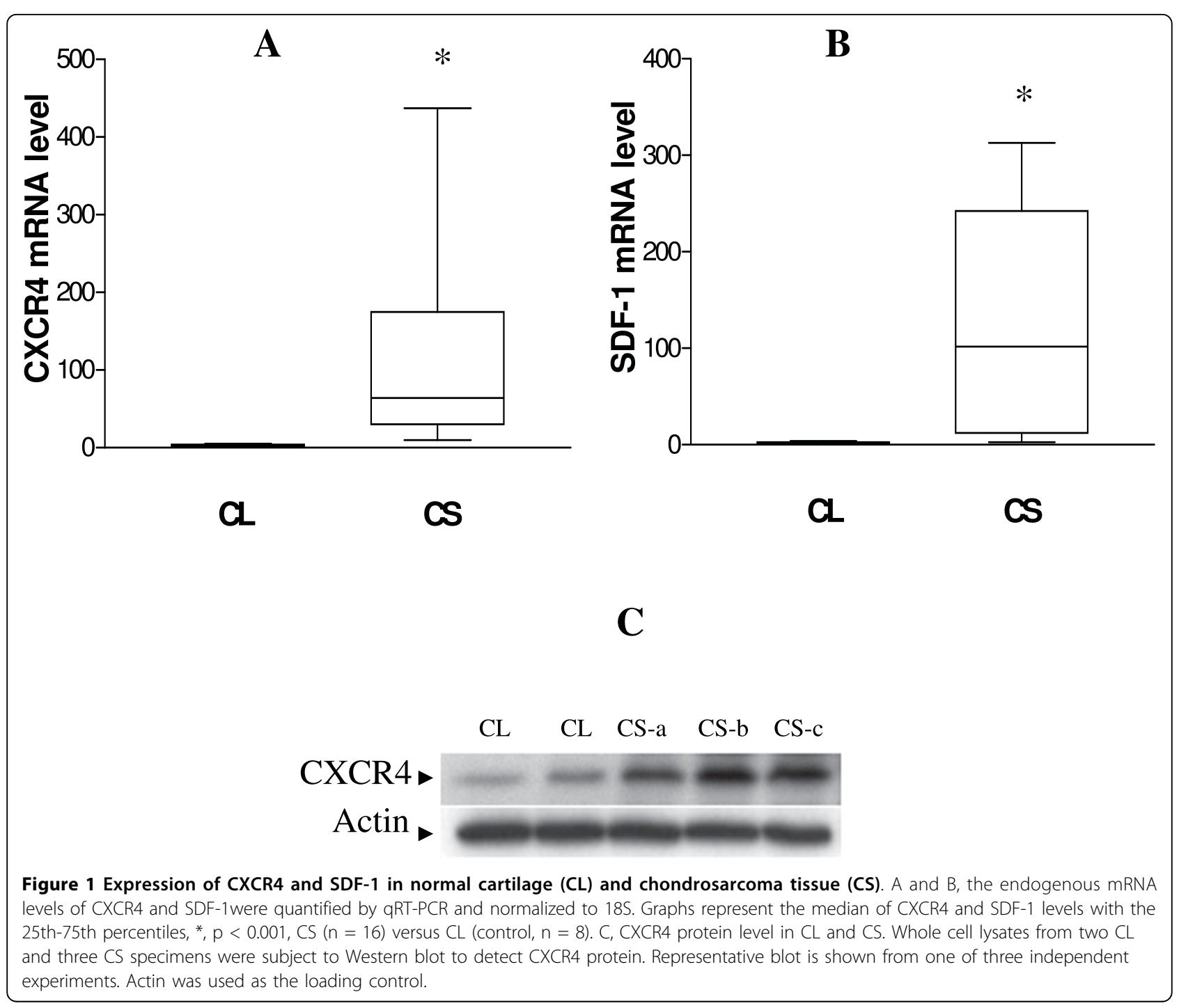

MMP1 mRNA expression 9 fold which was further increased to 23 fold by SDF1 stimulation. There was no effect of SDF1 or AMD3100 during normoxia on MMP1 mRNA level. AMD3100 blocked the SDF1 mediated increase in MMP1 mRNA during hypoxia (Fig 6A). Similarly, hypoxia and SDF1 increased active MMP1 in conditioned media of cells cultured in hypoxia. AMD3100 had no effect during hypoxia without SDF1. AMD3100 in the presence of SDF1 had a similar effect as the MMP inhibitor O-phenanthroline (Fig 6B).

Downstream effects of hypoxia and CXCR4/SDF-1 are mediated through ERK signaling

In order to assess the role of MAP kinases in CXCR4/ SDF1 signaling, time course analysis of MAP kinase expression after SDF1 exposure was performed. SDF1 stimulation during hypoxia transiently increased phosphorylated ERK which reached a peak at 10 minutes.
The increase in phosphorylated ERK could be inhibited by MEK (ERK) inhibitor U0126 (Fig. 7A). There was less effect of SDF1 on phosphorylated JNK and no effect on p38. SDF1 stimulation during hypoxia also increased MMP1 protein expression. Both the CXCR4 inhibitor AMD3100, the ERK inhibitor U0126, and ERK1/2 siRNA inhibited MMP1 protein expression (Fig. 7B, Additional file 1). The SDF1 mediated increase in cell invasion during hypoxia was also inhibited by U0126 and ERK1/2 siRNA, but not by the other MAP kinase inhibitors SP600125 and SB203580 (Fig 7C, D, Additional file 1).

\section{Discussion}

A better understanding of the mechanisms underlying invasive behavior of a cancer is an important first step in developing improved treatment strategies. This study provides the first indication that CXCR4 is regulated by 


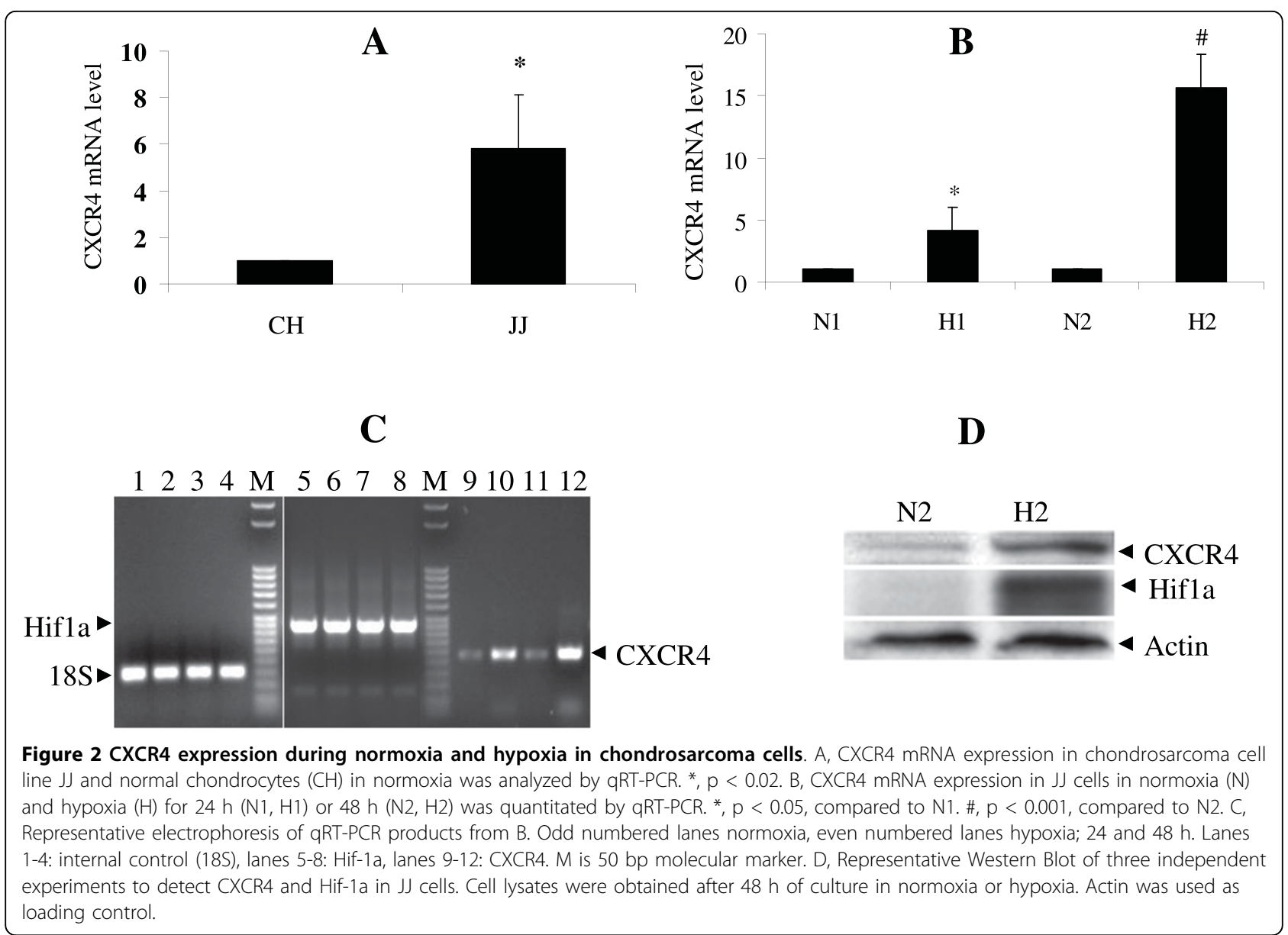

hypoxia and specifically HIF-1a in chondrosarcoma cells. We also show that increased CXCR4 signaling regulates expression of MMP1, a factor known to be involved with chondrosarcoma metastasis and a marker for poor prognosis[11]. Overexpression of CXCR4 has been reported in a variety of tumors, primarily carcinoma. In carcinoma, CXCR4 expression mediates metastasis to bone, which has relatively high levels of SDF1. In chondrosarcoma, it is possible that local SDF1 stimulates local tumor growth in a paracrine manner, and for those cells which gain access to the circulation, may also partially account for the tendency of these tumors to develop lung metastases, since the lung also contains high levels of SDF1. Factors such as MMP1 mediate local migration out of the microenvironment, ie stroma for carcinoma and bone for chondrosarcoma, and into the circulation. Factors such as CXCR4 mediate homing and growth at distant sites. Within sarcoma, CXCR4 expression has been detected in osteosarcoma $[6,12]$ and recently in chondrosarcoma[13]. Our results confirm the expression of CXCR4 in both chondrosarcoma tissue and cell lines and also show that CXCR4 expression was higher in high grade tumors, that hypoxia and HIF-1a enhance CXCR4/SDF1 mediated invasion through upregulation of CXCR4 expression, and that CXCR4/SDF1 signaling increases invasion through ERK mediated increase in MMP1 expression and activity.

Hypoxia, primarily acting through HIF-1a, elicits a wide spectrum of changes in gene expression that contribute to the metastatic phenotype of cancer cells. Hypoxia and Hif-1a have been shown to upregulate CXCR4 in carcinomas such as lung cancer [14], oral squamous cell carcinoma[15], breast carcinoma [16], and renal cell carcinoma [17]. The mechanism of Hif-1a regulation of CXCR4 is through direct binding to the CXCR4 promoter [18]. Our results show that HIF-1a also upregulates CXCR4 in chondrosarcoma. Interestingly, during chondrogenic differentiation CXCR4 is downregulated[19]. Although chondrosarcoma share some markers of the cartilage phenotype, as cells become malignant, some repressed genes will be reexpressed. CXCR4 has been shown to be involved with cell migration and invasion in many systems. The data include in vitro invasion and migration assays as well as xenograft models of metastatic disease in which blockade of CXCR4 with drugs, peptides, or antibodies can 


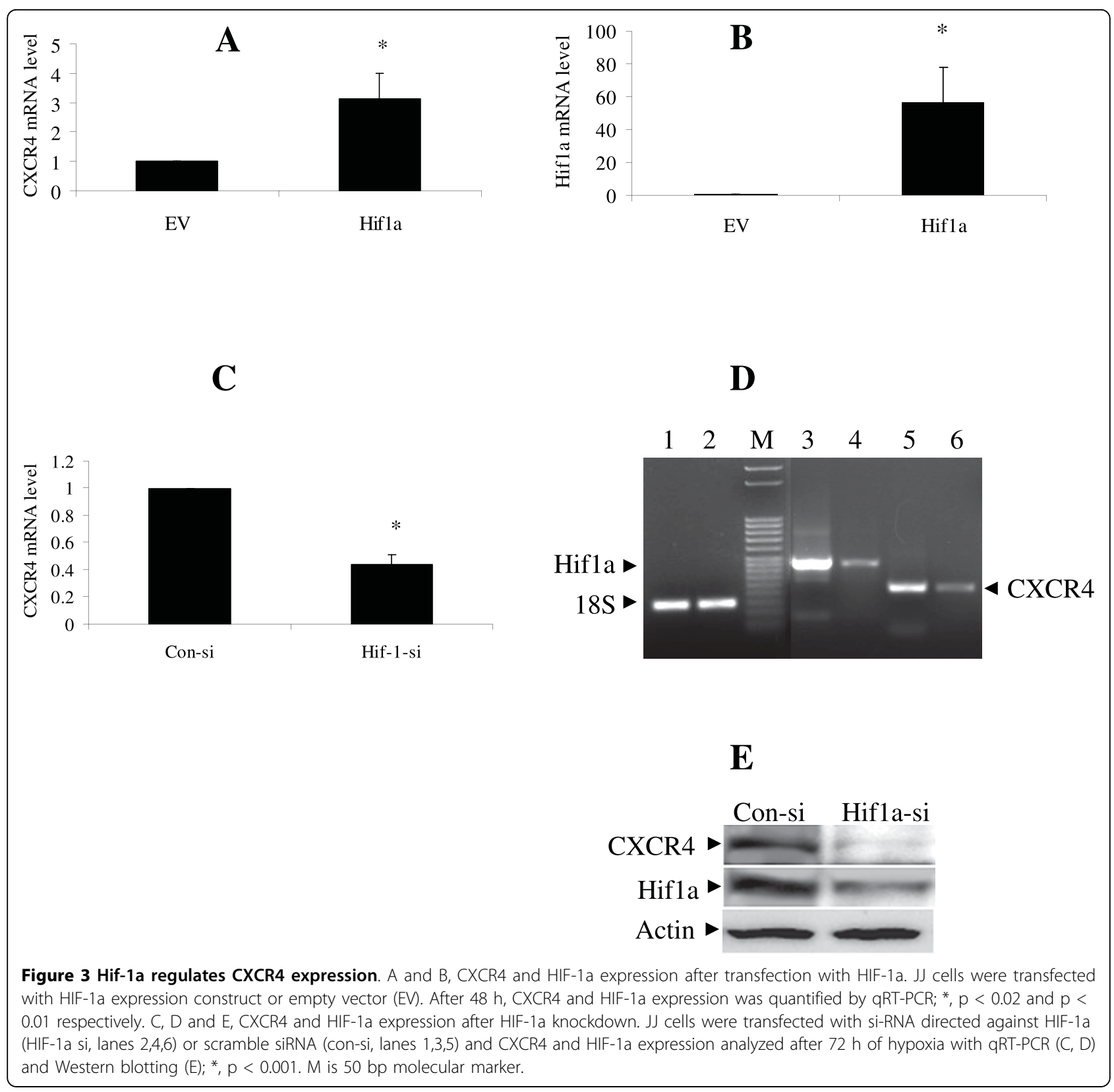

inhibit development and growth of metastases. Independent of CXCR4, MMP1 has also been shown to be involved with tissue invasion and development of metastases. MMP1 is also known to be upregulated by hypoxia and HIF-1a in breast and lung cancer cells $[20,21]$, and also by CXCR4 in Nk cells [22] and prostate cancer cells [23]. However, this project is the first to link the combined effects of HIF-1a on CXCR4 and MMP1 expression and the indirect effect of HIF-1a on MMP1 expression acting through CXCR4, which independently increases MMP1 in chondrosarcoma cells.

The role of MMP1 in chondrosarcoma invasion and its role as a poor prognostic indicator have been known for some time $[11,24]$. Inhibition of MMP1 with siRNA has been shown to decrease chondrosarcoma cell invasion [25-27]. We have shown that one mechanism of increased MMP1 in chondrosarcoma is mediated through CXCR4 signaling, which is amplified by hypoxia, and is mediated by ERK, but not other MAP kinases. siRNA directed against HIF-1a, CXCR4, ERK; CXCR4 blockade with AMD3100; or ERK inhibitor U0126 all efficiently inhibited the increase in invasion of chondrosarcoma cells during hypoxia. A previous study of CXCR4 in chondrosarcoma invasion during normoxia showed that CXCR signaling increased expression of alphavbeta3 integrin, also through ERK, and that 


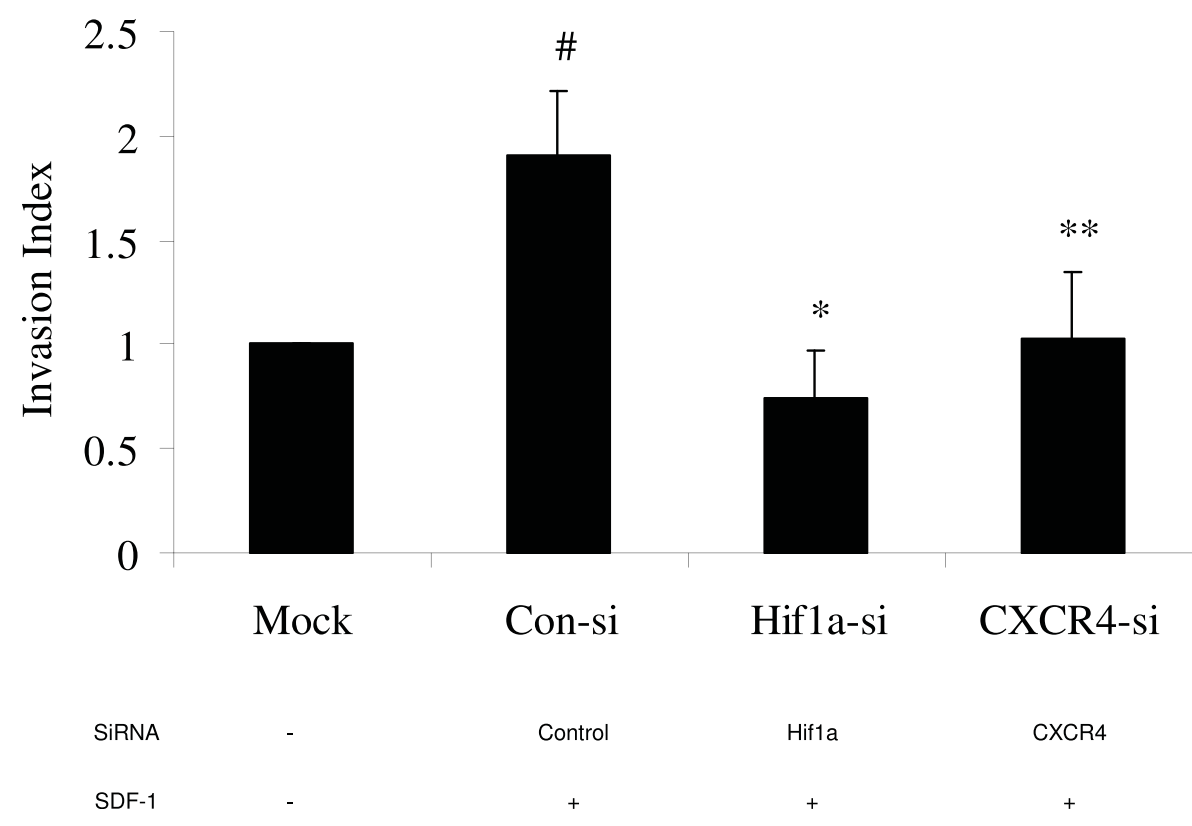

Figure 4 Effect of Hif-1a and CXCR4 knockdown on the invasion of chondrosarcoma cells. JJ cells were cultured in normoxia (mock) or hypoxia for $48 \mathrm{~h}$ after transfection with scramble siRNA (Con-si), or siRNA directed against Hif-1a or CXCR4. Invasion assay was then performed as described in Methods. \#, p $<0.05$, compared to Mock; ${ }^{*}, p<0.01,{ }^{* *}, p<0.05$, compared with Con-si.

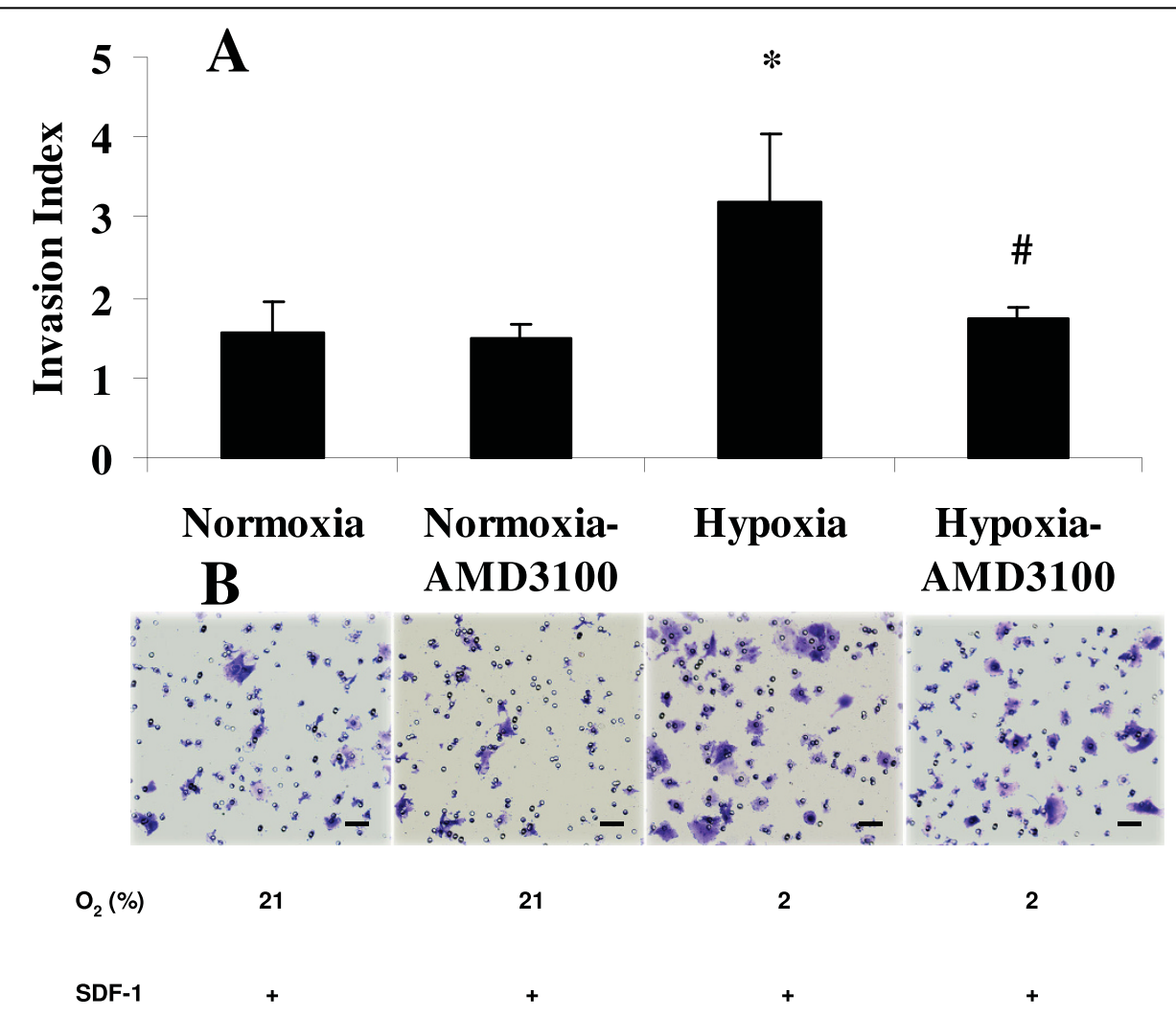

Figure 5 Effect of hypoxia and CXCR4 inhibition on the invasion of chondrosarcoma cells. JJ cells were cultured under normoxia or in hypoxia with $10 \mathrm{ng} / \mathrm{ml} \mathrm{SDF1}$ in the presence or absence of $0.1 \mu \mathrm{M}$ of AMD3100 for $48 \mathrm{~h}$ (AMD3100 was added $2 \mathrm{~h}$ before SDF1) before performing the invasion assay, which was also performed during normoxia or hypoxia with or without AMD3100. *, $p<0.01 ; \#, p<0.05$ compared to Hypoxia. Upper panel, bar graphs represent data from three independent experiments. Lower panel, representative photographs of stained cells on the lower side of matrigel membrane from one of three experiments. Original magnifications, 100x. 

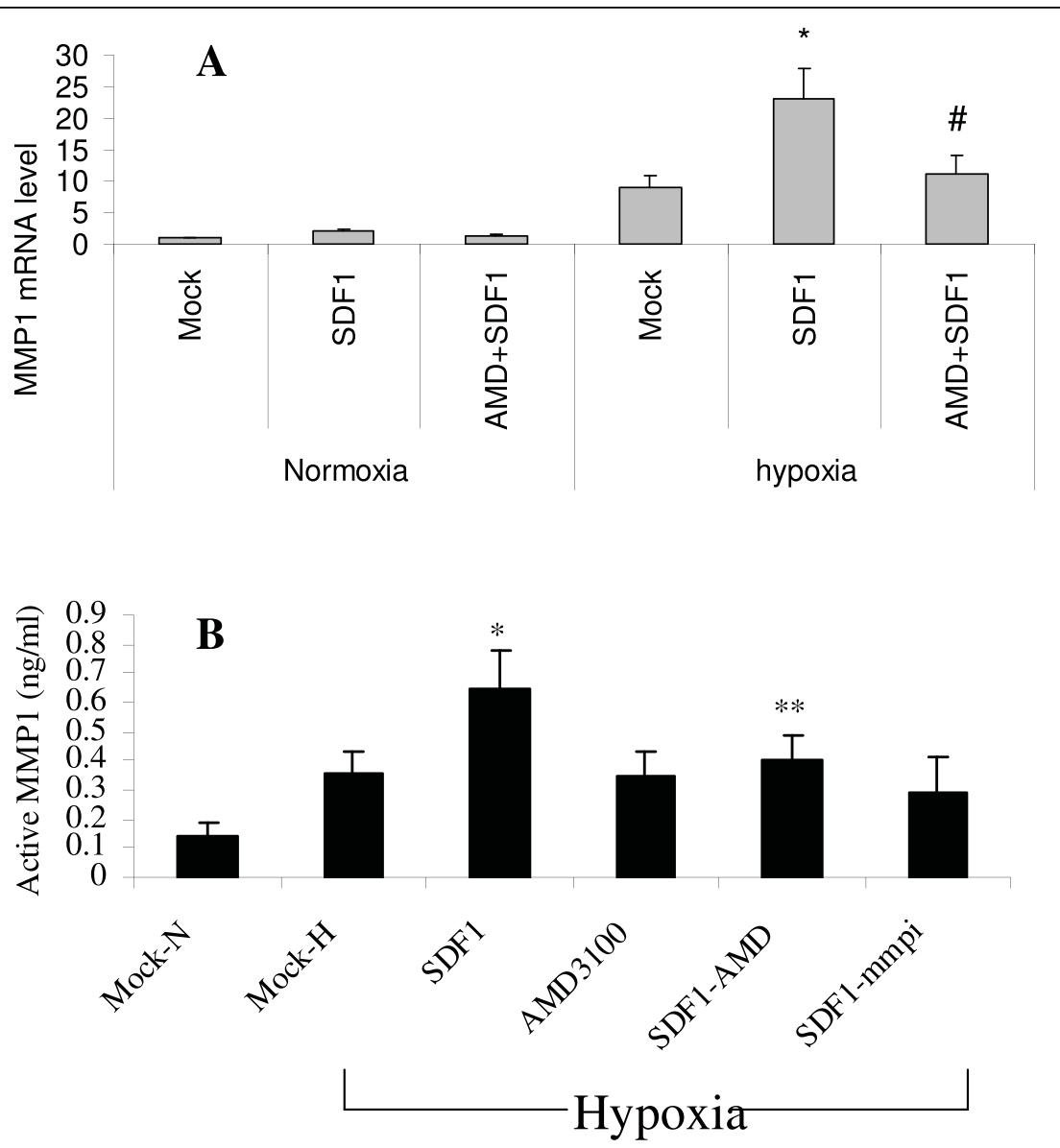

Figure 6 SDF1 increases MMP1 mRNA level and MMP1 activity during hypoxia. Tumor cells were cultured on matrigel with only medium (MOCK), SDF1 $(10 \mathrm{ng} / \mathrm{ml})$, AMD3100 $0.1 \mu \mathrm{M}$, or SDF1+AMD3100 (AMD3100 was added $2 \mathrm{~h}$ before SDF1), or SDF1 + mmpi (MMPs inhibitor Ophenanthroline, $10 \mathrm{nM}$ ) for $24 \mathrm{~h}$. Conditioned medium was then collected for ELISA and cells were harvested for RNA isolation. A, MMP-1 mRNA level was measured by qRT-PCR (*, $p<0.001$, compared to Mock in hypoxia; $\#, p<0.001$, compared to SDF1 in hypoxia). B, active MMP-1 was measured in conditioned media by ELISA (*, $\mathrm{p}<0.01$, compared to Mock-H in hypoxia; ${ }^{* *}, \mathrm{p}<0.05$, compared to SDF1 in hypoxia).

alphavbeta3 integrin antibodies could also inhibit chondrosarcoma invasion in vitro[13]. Therefore, CXCR4 affects chondrosarcoma invasion through upregulation of multiple genes including alphavbeta 3 integrin and MMP1. In other tumors and chondrosarcoma (Additional file 2), CXCR4 signaling upregulates other MMPs such as MMP-2, 8 and -9 and -13[28,29]. Since CXCR signaling upregulates multiple genes related to metastasis and since clinical MMP inhibition is not currently feasible, whereas CXCR4 blockade is possible with drugs such as AMD3100, CXCR4 may be a fruitful therapeutic target to inhibit some of the metastatic potential of chondrosarcoma cells.

\section{Conclusions}

We present data that shows hypoxia mediated increase in MMP1 expression and chondrosarcoma invasion is partially mediated by CXCR4 signaling. CXCR4 blockade can inhibit the effects of hypoxia on MMP1 expression and chondrosarcoma invasion in vitro, suggesting that CXCR4 blockade could be a therapeutic target to inhibit chondrosarcoma invasion and metastasis. The effectiveness of this strategy requires in vivo confirmation.

\section{Methods}

Tissue

Articular cartilage, chondrosarcoma tissue, and cancellous bone were obtained from surgical specimens, and either preserved in RNAlater Solution (Applied Biosystems, Foster City, CA) or snap frozen in liquid nitrogen for later use. There were 8 articular cartilage specimens and 16 chondrosarcoma ( 3 grade I, 5 grade II, and 8 grade III). IRB approval was obtained.

Cell lines and cell culture

Human chondrocytes isolated from normal adult articular cartilage and chondrosarcoma (CS) cell line JJ (a gift from Dr. Joel Block, Rush Medical School, Chicago, IL) 


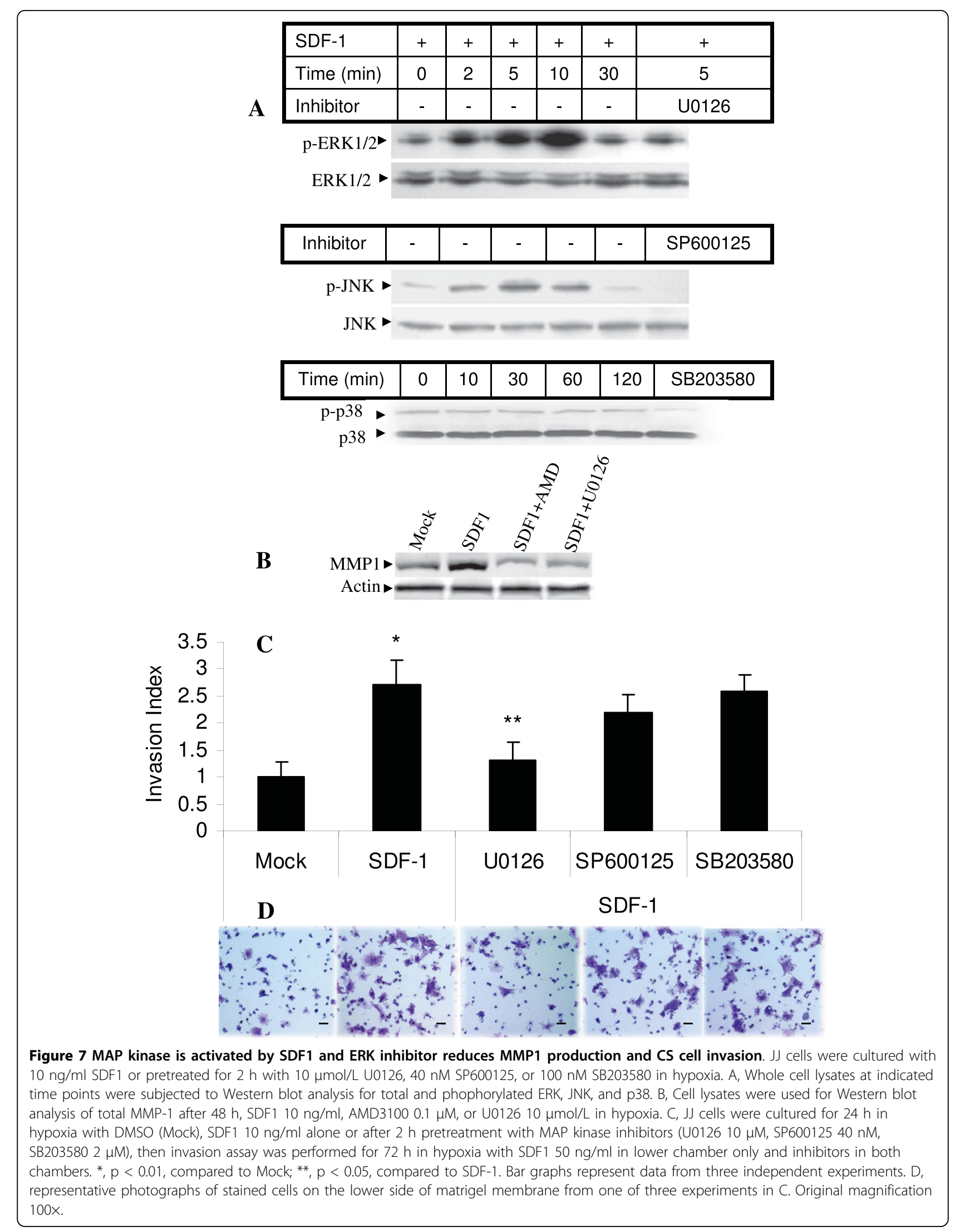


were cultured in complete medium (40\% DMEM, 40\% MEM, 20\% F12) with 10\% FBS. All cells were cultured in a humidified incubator (NuAire Inc, Plymouth $\mathrm{MN}$ ) under $5 \% \mathrm{CO}_{2}$ and either normoxia (ambient oxygen) or hypoxia $\left(2 \% \mathrm{O}_{2}\right)$ [30]. JJ was derived from a human grade II chondrosarcoma[31]. The drugs and inhibitors used were: AMD3100 (Sigma Chemical Company St. Louis, MO), human recombinant SDF-1 (R\&D Systems, Minneapolis, MN), MMP inhibitor O-phenanthroline (Sigma), MAP kinase inhibitors: MEK1/2 inhibitor U0126 (Cell Signaling, Beverly, MA), JNK inhibitor SP600125 (A.G. Scientific Inc., San Diego, CA), p38 inhibitor SB203580 (BioMol International, Plymouth Meeting, PA) or DMSO (Sigma), solvent for the inhibitors.

\section{Transfections}

Cells were transiently transfected with an expression construct for human Hif-1a in pcDNA3.1(+) vector (Invitrogen, Carlsbad, CA), (a gift from Hirouki Kato PhD, Tokyo Metropolitan Institute of Medical Science, Tokyo, Japan), or empty vector using Fugene HD (Roche, Indianapolis, IN) in 6 or 12 well plates $24 \mathrm{~h}$ after seeding. Cells were then incubated for $48 \mathrm{~h}$ and harvested for the following experiments.

\section{RNA interference (siRNA)}

Cells were transfected with Hif-1a siRNA (Qiagen, Valencia, CA), CXCR4 siRNA (Dharmacon, Lafayette, CO), ERK1/2 siRNA(Cell Signaling Technology, Inc., Beverly, MA) or control siRNA by HiPerFect transfection reagent (Qiagen). RNA and protein were obtained $72 \mathrm{~h}$ after transfection for qRT-PCR and Western Blot analysis.

\section{Real-time RT-PCR (qPCR)}

RNA was isolated from cells with RNAqueous Kit (Ambion, Austin, TX) or tissues with Trizol Reagent (Invitrogen). After treatment with TURBO DNase (Ambion), one microgram of RNA was reverse transcribed with random hexamers to obtain first-strand cDNA using iScript cDNA kit (Bio-Rad, Philadelphia, PA). The quantification of mRNA for Hif-1a, CXCR4, SDF-1, and MMP1 was performed by two-step real-time quantitative RT-PCR (Qiagen). Primers (5'-3') for Hif-1a were: forward, ctc aaa gtc gga cag cct ca; reverse, ccc tgc agt agg ttt ctg ct; for CXCR4, forward: gtc cac gcc acc aac ag, reverse: ctg ttg gtg gcg tgg ac; for SDF-1, forward: cgt gct ggt cct cgt gct gac; reverse: gct ttc tcc agg tac tcc tg; for MMP1, forward: gag caa aca cat ctg acc tac agg a; and reverse, ttg tcc cga tga tct ccc ctg aca; $18 \mathrm{~S}$ was used as an internal control since it has been shown to be the optimal reference gene[32]. Amplification conditions were as follows: 2 min preincubation at $50^{\circ} \mathrm{C}, 10$ minutes at $95^{\circ} \mathrm{C}$ for enzyme activation, and 40 cycles at $95^{\circ} \mathrm{C}$ denaturation for $10 \mathrm{~s}, 55^{\circ} \mathrm{C}$ annealing for $30 \mathrm{~s}\left(59^{\circ} \mathrm{C}\right.$ for MMP1) and $72^{\circ} \mathrm{C}$ extension for $30 \mathrm{~s}$. The comparative threshold cycle $(\mathrm{Ct})$ method, i.e., $2^{-\Delta \Delta \mathrm{Ct}}$ method was used for the calculation of fold amplification[33]. Each experiment was evaluated with three PCR reactions and each experiment was repeated three times. Data are presented as mean value \pm SD.

\section{Western Blot Analysis}

Protein from cell lysates of tissues or cells were separated via SDS-PAGE and probed with antibodies for CXCR4, MMP1, actin (Santa Cruz Biotechnology, Santa Cruz, CA); and antibodies for Hif-1a, p-ERK, ERK, pJNK, JNK, p-p38, and p38 (Cell Signaling Technology, Inc.). Western Blot analyses were performed as previously described [10]. Protein concentrations were determined using the Bio-Rad Quick Start Bradford protein assay (Bio-Rad, Hercules, CA) and the equivalent of forty $\mu \mathrm{g}$ of protein were subjected to SDS-PAGE (Lonza Inc., Allendale, NJ).

\section{ELISA Assay}

After treatment, cells were cultured $\mathrm{O} / \mathrm{N}$ in $\mathrm{FBS}$ free medium and the conditioned media (CM) from CS cells was concentrated using Centricon-30 centrifugal filter device (Millipore, Billerica, MA). The amount of active MMP1 was detected using Human Active MMP1 Fluorescent Assay kit (R\&D Systems, Minneapolis, MN) according to the manufacturer's instructions. Active MMP1 in the CM was measured in duplicate for each sample and normalized to the cell number at the end of the culture period. Each experiment was repeated three times.

\section{Tumor cell invasion assay}

Invasive activity of CS cells was analyzed with matrigel coated BD Falcon Cell Culture Inserts (BD Biosciences, Bedford, MA). Briefly, $180 \mu \mathrm{l}$ of BD Matrigel Matrix Growth Factor Reduced (BD Biosciences, Bedford, MA) diluted 1:3 with serum-free medium was used to coat 8 $\mu \mathrm{m}$ pore size 12 -well inserts and incubated at $37^{\circ} \mathrm{C}$ for 2 h. After various treatments, during which cells are cultured $\mathrm{O} / \mathrm{N}$ without FBS, the cells were harvested by trypsinization, counted, and resuspended in complete medium containing $1 \%$ FBS at a concentration of $10^{6} /$ ml. $800 \mu$ containing $8 \times 10^{5}$ cells were added to each of the upper wells. $1.5 \mathrm{ml}$ of $5 \%$ FBS complete medium containing recombinant SDF1 (50 ng/ml, R\&D Systems) was added to the lower wells. After incubating for $72 \mathrm{~h}$ in hypoxia, cells that had invaded across the membrane were stained with Cell Stain Solution (Millipore), washed, photographed, then lysed and cell number quantitated by absorbance at $560 \mathrm{~nm}$ on a standard microplate reader. The invasion index was calculated by normalizing to the number of cells invading when the lower well has no SDF1 or FBS.

\section{Statistics}

All the experiments were repeated at least 3 times. Statistical analysis was performed with GraphPad Prism, v 
3.0 (GraphPad Software, San Diego, CA). ELISA results and CXCR4 expression in different grades of chondrosarcoma were analyzed with one-way ANOVA. Post test comparisons were made with Bonferroni correction. Experiments with two groups were analyzed with the Student's t-test. The null hypothesis of no difference was rejected at a significance level of $5 \%$.

Additional file 1: Effect of ERK knockdown on MMP1 expression and invasion in chondrosarcoma cells. A, Whole cell lysates from J cells were used for Western blot analysis of total MMP-1 after $48 \mathrm{~h}$ in hypoxia after ERK siRNA transfection. B, JJ cells were transfected with ERK siRNA. After 48 hours in hypoxia, invasion assay was performed as described in Methods.

Click here for file

[http://www.biomedcentral.com/content/supplementary/1476-4598-9-17S1.PPT ]

Additional file 2: MMPs expressed in chondrosarcoma cells. JJ cells were cultured in hypoxia $48 \mathrm{~h}$ without (mock) or with SDF-1 for 2 days. ${ }^{*}, p<0.02,{ }^{* *}, p<0.03, \#, p<0.04$

Click here for file

[ http://www.biomedcentral.com/content/supplementary/1476-4598-9-17S2.PPT]

\section{Acknowledgements}

The project described was supported by Grant Number 1P20RR024484-01, from the National Center for Research Resources (NCRR), a component of the National Institutes of Health $(\mathrm{NIH})$ and its contents are solely the responsibility of the authors and do not necessarily represent the official view of NCRR or NIH. This work was also supported by the RIH Orthopaedic Foundation. RMT thanks Jack Wands, MD for advice and mentorship This paper was presented in abstract form at the $54^{\text {th }}$ Annual Meeting of the Orthopaedic Research Society, San Francisco, 2008.

\section{Authors' contributions}

XS participated in the formation of the hypotheses, carried out the molecular biology experiments, and participated in interpretation of the data.

RMT participated in the formation of the hypotheses, in interpretation of the data, and drafted the manuscript.

QC participated in the formation of the hypotheses and in interpretation of the data.

LW participated in the formation of the hypotheses and in interpretation of the data.

All authors read and approved the final manuscript.

\section{Competing interests}

The authors declare that they have no competing interests.

Received: 11 September 2009

Accepted: 26 January 2010 Published: 26 January 2010

\section{References}

1. Soderstrom M, Ekfors TO, Bohling TO, Teppo LH, Vuorio El, Aro HT: No improvement in the overall survival of 194 patients with chondrosarcoma in Finland in 1971-1990. Acta Orthop Scand 2003, 74:344-350.

2. Giuffrida AY, Burgueno JE, Koniaris LG, Gutierrez JC, Duncan R, Scully SP: Chondrosarcoma in the United States (1973 to 2003): an analysis of 2890 cases from the SEER database. J Bone Joint Surg Am 2009, 91:1063-1072

3. Lee FY, Mankin HJ, Fondren G, Gebhardt MC, Springfield DS, Rosenberg AE, Jennings LC: Chondrosarcoma of bone: an assessment of outcome. J Bone Joint Surg Am 1999, 81:326-338.
4. Muller A, Homey B, Soto H, Ge N, Catron D, Buchanan ME, McClanahan T, Murphy E, Yuan W, Wagner SN, Barrera JL, Mohar A, Verastegui E, Zlotnik A: Involvement of chemokine receptors in breast cancer metastasis. Nature 2001, 410:50-56.

5. Wang J, Wang J, Sun Y, Song W, Nor JE, Wang CY, Taichman RS: Diverse signaling pathways through the SDF-1/CXCR4 chemokine axis in prostate cancer cell lines leads to altered patterns of cytokine secretion and angiogenesis. Cell Signal 2005, 17:1578-1592.

6. Laverdiere C, Hoang BH, Yang R, Sowers R, Qin J, Meyers PA, Huvos AG, Healey $\mathrm{JH}$, Gorlick R: Messenger RNA expression levels of CXCR4 correlate with metastatic behavior and outcome in patients with osteosarcoma. Clin Cancer Res 2005, 11:2561-2567.

7. Raman D, Baugher PJ, Thu YM, Richmond A: Role of chemokines in tumor growth. Cancer Lett 2007, 256:137-165.

8. Darash-Yahana M, Pikarsky E, Abramovitch R, Zeira E, Pal B, Karplus R, Beider K, Avniel S, Kasem S, Galun E, Peled A: Role of high expression levels of CXCR4 in tumor growth, vascularization, and metastasis. FASEB J 2004, 18:1240-1242

9. McGough RL, Lin C, Meitner P, Aswad BI, Terek RM: Angiogenic cytokines in cartilage tumors. Clin Orthop Relat Res 2002, 62-69.

10. Schioppa T, Uranchimeg B, Saccani A, Biswas SK, Doni A, Rapisarda A, Bernasconi S, Saccani S, Nebuloni M, Vago L, Mantovani A, Melillo G, Sica A: Regulation of the chemokine receptor CXCR4 by hypoxia. J Exp Med 2003, 198:1391-1402.

11. Berend KR, Toth AP, Harrelson JM, Layfield LJ, Hey LA, Scully SP: Association between ratio of matrix metalloproteinase- 1 to tissue inhibitor of metalloproteinase- 1 and local recurrence, metastasis, and survival in human chondrosarcoma. J Bone Joint Surg Am 1998, 80:11-17.

12. Perissinotto E, Cavalloni G, Leone F, Fonsato V, Mitola S, Grignani G, Surrenti N, Sangiolo D, Bussolino F, Piacibello W, Aglietta M: Involvement of chemokine receptor 4/stromal cell-derived factor 1 system during osteosarcoma tumor progression. Clin Cancer Res 2005, 11:490-497.

13. Lai TH, Fong YC, Fu WM, Yang RS, Tang CH: Stromal cell-derived factor-1 increase alphavbeta3 integrin expression and invasion in human chondrosarcoma cells. J Cell Physiol 2009, 218:334-342

14. Phillips RJ, Mestas J, Gharaee-Kermani M, Burdick MD, Sica A, Belperio JA, Keane MP, Strieter RM: Epidermal growth factor and hypoxia-induced expression of CXC chemokine receptor 4 on non-small cell lung cancer cells is regulated by the phosphatidylinositol 3-kinase/PTEN/AKT/ mammalian target of rapamycin signaling pathway and activation of hypoxia inducible factor-1alpha. J Biol Chem 2005, 280:22473-22481.

15. Ishikawa T, Nakashiro K, Klosek SK, Goda H, Hara S, Uchida D, Hamakawa H: Hypoxia enhances CXCR4 expression by activating HIF-1 in oral squamous cell carcinoma. Oncol Rep 2009, 21:707-712

16. Shim H, Lau SK, Devi S, Yoon Y, Cho HT, Liang Z: Lower expression of CXCR4 in lymph node metastases than in primary breast cancers: potential regulation by ligand-dependent degradation and HIF-1alpha. Biochem Biophys Res Commun 2006, 346:252-258.

17. Pan J, Mestas J, Burdick MD, Phillips RJ, Thomas GV, Reckamp K, Belperio JA Strieter RM: Stromal derived factor-1 (SDF-1/CXCL12) and CXCR4 in renal cell carcinoma metastasis. Mol Cancer 2006, 5:56.

18. Dunn LK, Mohammad KS, Fournier PG, McKenna CR, Davis HW, Niewolna M, Peng XH, Chirgwin JM, Guise TA: Hypoxia and TGF-beta drive breast cancer bone metastases through parallel signaling pathways in tumor cells and the bone microenvironment. PLoS One 2009, 4:e6896.

19. Cristino S, Piacentini A, Manferdini C, Codeluppi K, Grassi F, Facchini A, Lisignoli G: Expression of CXC chemokines and their receptors is modulated during chondrogenic differentiation of human mesenchymal stem cells grown in three-dimensional scaffold: evidence in native cartilage. Tissue Eng Part A 2008, 14:97-105.

20. Fu OY, Hou MF, Yang SF, Huang SC, Lee WY: Cobalt chloride-induced hypoxia modulates the invasive potential and matrix metalloproteinases of primary and metastatic breast cancer cells. Anticancer Res 2009, 29:3131-3138.

21. Shyu KG, Hsu FL, Wang MJ, Wang BW, Lin S: Hypoxia-inducible factor 1alpha regulates lung adenocarcinoma cell invasion. Exp Cell Res 2007, 313:1181-1191.

22. Goda $S$, Inoue H, Umehara H, Miyaji M, Nagano $Y$, Harakawa N, Imai H, Lee $P$, Macarthy JB, Ikeo T, Domae N, Shimizu Y, lida J: Matrix metalloproteinase-1 produced by human CXCL12-stimulated natural killer cells. Am J Pathol 2006, 169:445-458. 
23. Singh S, Singh UP, Grizzle WE, Lillard JW Jr: CXCL12-CXCR4 interactions modulate prostate cancer cell migration, metalloproteinase expression and invasion. Lab Invest 2004, 84:1666-1676.

24. Jiang X, Dutton CM, Qi W, Block JA, Brodt P, Durko M, Scully SP: Inhibition of MMP-1 expression by antisense RNA decreases invasiveness of human chondrosarcoma. J Orthop Res 2003, 21:1063-1070.

25. Yuan J, Dutton CM, Scully SP: RNAi mediated MMP-1 silencing inhibits human chondrosarcoma invasion. J Orthop Res 2005, 23:1467-1474.

26. Jiang X, Dutton CM, Qi WN, Block JA, Garamszegi N, Scully SP: siRNA mediated inhibition of MMP-1 reduces invasive potential of a human chondrosarcoma cell line. J Cell Physiol 2005, 202:723-730.

27. Jiang X, Dutton CM, Qi W, Block JA, Brodt P, Durko M, Scully SP: Inhibition of MMP-1 expression by antisense RNA decreases invasiveness of human chondrosarcoma. J Orthop Res 2003, 21:1063-1070.

28. Shen $X$, Wang $S$, Wang $H$, Liang M, Xiao L, Wang Z: The role of SDF-1/ CXCR4 axis in ovarian cancer metastasis. J Huazhong Univ Sci Technolog Med Sci 2009, 29:363-367.

29. Tan CT, Chu CY, Lu YC, Chang CC, Lin BR, Wu HH, Liu HL, Cha ST, Prakash E, Ko JY, Kuo ML: CXCL12/CXCR4 promotes laryngeal and hypopharyngeal squamous cell carcinoma metastasis through MMP-13-dependent invasion via the ERK1/2/AP-1 pathway. Carcinogenesis 2008, 29:1519-1527.

30. Lin C, McGough R, Aswad B, Block JA, Terek R: Hypoxia induces HIF-1alpha and VEGF expression in chondrosarcoma cells and chondrocytes. $J$ Orthop Res 2004, 22:1175-1181.

31. Block JA, Inerot SE, Gitelis S, Kimura JH: Synthesis of chondrocytic keratan sulphate-containing proteoglycans by human chondrosarcoma cells in long-term culture. JBJS 1991, 73-A:647-658.

32. Asp J, Brantsing C, Lovstedt K, Benassi MS, Inerot S, Gamberi G, Picci P, Lindahl A: Evaluation of p16 and Id 1 status and endogenous reference genes in human chondrosarcoma by real-time PCR. Int J Oncol 2005, 27:1577-1582

33. Livak KJ, Schmittgen TD: Analysis of relative gene expression data using real-time quantitative PCR and the 2(-Delta Delta C(T)) Method. Methods 2001, 25:402-408.

doi:10.1186/1476-4598-9-17

Cite this article as: Sun et al:: CXCR4/SDF1 mediate hypoxia induced chondrosarcoma cell invasion through ERK signaling and increased MMP1 expression. Molecular Cancer 2010 9:17.

\section{Submit your next manuscript to BioMed Central and take full advantage of:}

- Convenient online submission

- Thorough peer review

- No space constraints or color figure charges

- Immediate publication on acceptance

- Inclusion in PubMed, CAS, Scopus and Google Scholar

- Research which is freely available for redistribution

Submit your manuscript at www.biomedcentral.com/submit 